\title{
Subject Index Vol. 11, 1995
}

Abdominal surgery

-, cell activation Suppl. 215

-, complications Suppl. 215

-, host defense Suppl. 215

-, immmunology Suppl. 215

-, infection Suppl. 215

-, inflammatory response Suppl. 215

-, risk evaluation Suppl. 215 Acute phase proteins Suppl. 242 Adenocarcinoma 2174

Adhesions, abdominal wall 192 -, intestinal wall 192 Adrenal cancer 134

gland 142

-, operation strategy 142

- - technique 142

-, tumors 153

medullary hyperplasia 148 Aggressive fïbromatosis 2160 Aldosteronoma 134

Anal canal 4298

Anorectum, anatomy 4298

-, continence 4298

-, surgery 4298

Antireflux surgery 3224

Appendectomy 3258

-, complications 3262

-, laparoscopic 3258,3262

-, open 3262

-, present concept 3262

Arteries, therapeutic blockade 16

Autologous predeposit 4395

- $\quad$ transfusion 4395

Benign diseases, colon 3252 Bile duct injuries Suppl. 136

- $\quad$ - stones 3210

Biliary pancreatitis Suppl. 242 Blunt liver trauma Suppl. 122 Brachytherapy 2144

Carcinoid 1 6,1 23

- $\quad$ syndrome 123

Cholangitis Suppl. 242

Cholelithiasis, therapy 2171

Chronic duodenal ulcer 3232

Coloanal anastomosis 4369

Colon carcinomas 3252

Combined radio-/chemotherapy 2 133, 
2144 Compensating mechanisms 190 Continence, preserving procedures 4346 Cushing adenoma 134 Cystic duct 2171 Cytokines Suppl. 154

Desmoid tumor 2160 Diagnostic laparoscopy 3268 Diagnostics Suppl. 251 Double stapling anastomosis 3246 Douglas abscess 3258 Dysontogenetic liver cysts 185

Embolization 16

Endocrine glands, neoplasms 16

- $\quad$ tumors 112

Endoscopic papillotomy 3210

2 171 retrograde cholangiopancreatography

Epidemiology Suppl. 118 Epigastric pain 2174 ERCP Suppl. 242 Erythropoietin 4395

Excision, abdominoperineal 4376 -, mesorectal 4334 Extracorporeal shock wave 2171

Fundoplication 3224

Gastric carcinoma 2133 Gastrinoma 16 -, diagnostic 118 -, therapy 118

Gastroesophageal reflux disease 3224 Gastrointestinal carcinoids, palliative surgery 123

-, medical treatment 123

hemorrhage 2174

- malignancies 3268

GM-CSF Suppl. 234

Hemoclip treatment 182 Hemostasis Suppl. 124 Hepatic trauma Suppl. 148

- $\quad$-, historical notes Suppl. 111

Hiatal hernia 3224

High-dose-rate afterloading 2144

IFN- $\gamma$ Suppl. 234 IL·IO Suppl. 234

Immunoparalysis Suppl. 25

-, implications Suppl. 25

Immunosuppression Suppl. 29

Incidentaloma 134

Incontinence 4380

Inflammatory host response Suppl. 251

Inguinal hernia 3274

Insulinoma 16

-, diagnostic 118

-, therapy 118

Intraabdominal infection Suppl. 251

Intraoperative diagnostic methods 3203

- $\quad$ irradiation 2139

-, evaluation of method 2139

-, pancreatic cancer 2139

radiation therapy 2124,2144

radiotherapy 2 115, 2133 Intravenous iron substitution 4395 Iron replacement 190

Laparoscopic bile-duct surgery 3210

cholecystectomy 3203,3218

-, long-term follow-up 3218

colorectal surgery 3246 
deroofing 185

hernia repair 3274

intraoperative ultrasound 3203

repair of perforated ulcer 3241

resection of sigmoid colon and rectum 2178

sonography 3268

surgery 3232,3252

- $\quad$ triple-stapling technique 2178

Laparoscopically assisted rectal resection

4380 Liver injuries Suppl. 1 36, Suppl. 1 40, Suppl. 150

- $\quad$-, conservative treatment

Suppl. 140

-, late complications Suppl. 150

-, late results Suppl. 150

-, operative management

Suppl. 136

-, operative treatment Suppl. 140

regeneration in vitro Suppl. 154

transplantation 169 , Suppl. 148

-, complications 169

-, expert opinion Suppl. 160

-, infection 176

-, operative management 169

-, prophylaxis 176

-, results 169

-, risk factors 176

trauma Suppl. 1 18, Suppl. 1 22, Suppl. 130

-, aetiopathogenesis Suppl. 122

-, biomechanics Suppl. 122

-, diagnostics Suppl. 130

-, expert opinion Suppl. 160

-, therapy Suppl. 130 Local recurrence 4334 Low anterior resection 4324

- -, complication rate 4324

-- , oncological criterions

4324

- $\quad$ - - , survival rate 4324

Methods of hemostasis Suppl. 124 Minimally invasive surgery 4380 Monitoring Suppl. 251 409

Monocyte deactivation Suppl. 234 Multimodality treatment 2 115, 2144 Multiple endocrine neoplasia types 1 and 2

128

clinical features 128

diagnostic tools 128 
screening 128

surgical therapy 128 Muscle transposition 4376

Neo-sphincter reconstruction 4376

Omental transposition flap 185

Pancreas 2160 -, neoplasms 16

secretion 112 Pancreatic cancer 2124

necrosis Suppl. 242 Pancreatitis Suppl. 226

-, HLA-DR expression on monocytes

Suppl. 226 -, immunomonitoring Suppl. 226 Paraganglioma 148 Parasacral surgery 4346

Partial hepatectomy Suppl. 154 Penetrating liver injury Suppl. 124

- -, complications Suppl. 124

- -, operation indication

Suppl. 124 Percutaneous endoscopic gastrostomy 2166

- -, aspiration etiology 2166

- -, enteral nutrition 2166

- -, gastrostomy adverse effects

2166

Peritonitis Suppl. 2 34, Suppl. 2 47, Suppl. 251

-, intensive monitoring Suppl. 247 -, procalcitonin (PCT) Suppl. 247 Phaeochromocytoma(s) 1 34,1 48,1 53 -, etiology 153 -, differential diagnosis 153 -, prognosis 153 Plasmapheresis

Suppl. 234 Postoperative anemia 1 90, 4395 Preoperative diagnostic methods 3203

Procalcitonin Suppl. 2 42, Suppl. 251

Radiotherapy, curative effects 2124 -, palliative effects 2124 Rectal adenoma 4380 - cancer 4 $314,4334,4340,4346,4351,4361,4369,4376$

anastomotic leakage 4361 continence 4361 diagnosis 4310

hand-sutured anastomosis 4361 local recurrence 4361 low anterior resection 4361 prognostic factors 4314 staging 4314 stenosis 4361 carcinoma 2 115, 2 144, 4380 resection 4 340,4 351 -, abdominoperineal 4340 -, indications 4340 -, sphincter preservation 4351

Rectum 4298

carcinoma, histopathological examination 4304

-, pathology 4304

-, surgical therapy 4304

- $\quad$-, tumor spread 4304

Resection, anterior 4334

Sepsis Suppl. 2 5, Suppl. 2 9, Suppl. 2 34, Suppl. 2 |51

-, surgical treatment Suppl. 29 Shouldice repair 3274 Small-bowel cancer 2174 Somatostatin 1 6 Stoma 4376

Stump insufficiency 3258 Supplemental sepsis therapy Suppl. 29 Surgical patients at risk

Suppl. 251 Surgical treatment 2174

Therapeutic endoscopy 182

splitting 3210 Therapeutical splitting 2203 TNF- $\alpha$ Suppl. 234 Transanal access 4380 Trigger

of transfusions 190 Tumor biology 112

marker 4310

staging 3268,4310

Ulcus formation 112

Ultrasonic diagnostics 192 
Upper gastrointestinal bleeding 182

Vagotomy 3232

410

Subject Index Vol. 11,1995 